\title{
Fractal Kinetic Behavior of Plasmin on the Surface
}

\section{of Fibrin Meshwork}

Funding Source Statement: This work was supported by the Hungarian Scientific Research Fund [OTKA 83023, 100753 and 112612].

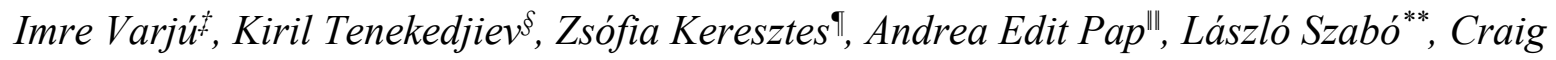
Thelwellt, Colin Longstafft, Raymund Machovich ${ }^{+}$, Krasimir Kolev t* $^{*}$

†Department of Medical Biochemistry, Semmelweis University, 1094 Budapest, Hungary; §Department of Information Technology, Nikola Vaptsarov Naval Academy, 9026 Varna, Bulgaria; ๆFunctional Interfaces Research Group, Institute of Materials and Environmental Chemistry, Research Centre for Natural Sciences, Hungarian Academy of Sciences, 1117 Budapest, Hungary; |||Microtechnology Department, Institute of Technical Physics and Materials

Science, Research Centre for Natural Sciences, Hungarian Academy of Sciences, 1121 Budapest, Hungary; **Department of Functional and Structural Materials, Institute of Materials and Environmental Chemistry, Research Centre for Natural Sciences, Hungarian Academy of Sciences, 1117 Budapest, Hungary; + Biotherapeutics Division, Haemostasis Section, National Institute for Biological Standards and Control, South Mimms, Herts, EN6 3QG UK

*Corresponding author: Semmelweis University, Department of Medical Biochemistry, 1094 Budapest, Tűzoltó u. 37-47., Hungary, tel.: +36 14591500/60035, fax: +36 1 2670031, email: Krasimir.Kolev@eok.sote.hu 


\section{ABBREVIATIONS}

AFM, atomic force microscope; $\varepsilon A C A, \varepsilon$-aminocaproic acid; FDP, fibrin degradation products;

SEM, scanning electron microscope; PBS, phosphate buffered saline; PDMS, poly(dimethylsiloxane). 


\section{ABSTRACT}

Intravascular fibrin clots are resolved by plasmin acting at the interface of gel-phase substrate and fluid-borne enzyme. The classic Michaelis-Menten kinetic scheme cannot describe satisfactorily this heterogeneous-phase proteolysis, because it assumes homogeneous well-mixed conditions. A more suitable model for these spatial constraints, known as fractal kinetics, includes a time-dependence of the Michaelis coefficient $K_{m}{ }^{F}=K_{m} 0^{F} .(1+t)^{h}$, where $h$ is a fractal exponent of time, $t$. The aim of the present study was to build up and experimentally validate a mathematical model for surface-acting plasmin that can contribute to a better understanding of the factors that influence fibrinolytic rates. The kinetic model was fitted to turbidimetric data for fibrinolysis under various conditions. The model predicted $K_{m 0}{ }^{F}=1.98 \mu \mathrm{M}$ and $h=0.25$ for fibrin composed of thin fibers and $K_{m 0}{ }^{F}=5.01 \mu \mathrm{M}$ and $h=0.16$ for thick fibers in line with a slower macroscale lytic rate (due to stronger clustering trend reflected in the $h$ value) despite faster cleavage of individual thin fibers (seen as lower $K_{m} 0^{F}$ ). $\varepsilon$-Aminocaproic acid at $1 \mathrm{mM}$ or $8 \mathrm{U} / \mathrm{ml}$ carboxypeptidase-B eliminated the time-dependence of $K_{m}{ }^{F}$ and increased the lysis rate suggesting a role of C-terminal lysines in the progressive clustering of plasmin. This fractal kinetic concept gained structural support from imaging techniques. Atomic force microscopy revealed significant changes in plasmin distribution on patterned fibrinogen surface in line with the time-dependent clustering of fluorescent plasminogen in confocal laser microscopy. These data from complementary approaches support a mechanism for loss of plasmin activity resulting from C-terminal lysine-dependent redistribution of enzyme molecules on fibrin surface. 
The scaffold of intravascular blood clots that are responsible for the acute tissue damage in ischemic cardio- and cerebrovascular disease is composed of fibrin meshwork and accordingly its dissolution is a primary strategy in the treatment of acute myocardial infarction and stroke $(1,2)$. This therapeutic approach is based on the administration of tissue-type (or other) plasminogen activator that attacks the clot from the circulating blood and binds to the surface of fibrin forming an interfacial layer, in which plasminogen is converted to plasmin, a protease capable of hydrolyzing the fibrin matrix to water-soluble degradation products (FDPs) (reviewed in 3). Even if plasmin is generated by fibrin-bound activator, it needs to detach in order to attack the susceptible cleavage sites in fibrin. Thus, a layer adjacent to the surface is formed, in which free plasmin is in equilibrium with the fibrin-bound enzyme engaged in catalytic action. As a result of fibrin break-down, this lytic front moves from the surface to the core of the clot and the rate of its migration characterizes the overall efficiency of thrombolysis $(4,5)$. Recently, direct plasmin application to the surface of thrombi has been proposed as a safe and efficient thrombolytic modality (reviewed in 6). Thus, independently of the plasmin source (local generation or bulk fluid phase-borne) all available enzyme-based approaches to resolve fibrin clots involve proteolytic events at the surface of a solid phase substrate. The optimization of the current thrombolytic agents and the design of new thrombolytics would greatly benefit from kinetic models that adequately treat this heterogeneous phase catalytic process. However, at present the treatment of fibrinolysis in enzymological terms lacks a model with parameters, which are mechanistically related to discrete steps of plasmin action.

The existing kinetic models of fibrinolysis fall largely into three categories. In certain cases (e.g. our earlier work 7) the global efficiency of proteases acting on fibrin could be characterized with phenomenological kinetic parameters that allow adequate comparison of different enzymes, 
but lack mechanistic content. A different approach builds upon diffusion, binding and catalytic data gained in separate experiments in attempt to reconstitute the global course of interfacial proteolysis from these discrete events $(8-10)$. In these models the discrete steps are mechanistically substantiated, but due to the complex nature of the examined phenomenon a high number of independently gained parameters are necessary, which inevitably leads to simplifications to account for the variability of the conditions, under which these are derived, and thus some essential mechanistic aspects can be overlooked. For example, although $80 \%$ of the fibrin fiber volume is occupied by water $(11,12)$, this space is highly compartmented by the protofibrils aligned in parallel within the fibers and by the higher ordering of the fibers encompassing three-dimensional pores. However, in environments with similar fixed geometric obstacles within cells, or in the extracellular matrix, anomalous diffusion is observed $(13,14)$. Thus, any model that is based on diffusion, binding and reaction rate parameters gained in homogeneous dilute solutions is necessarily just an approximation of the real situation in fibrin, where an additional level of complexity is rendered by the variability of fibrin meshwork structure (e.g. in cell-free areas of in vivo human thrombi the fiber diameter varies in the range $100-200 \mathrm{~nm}$ and the pore diameter in the range $160-380 \mathrm{~nm})(15)$. The third type of kinetic models of interfacial proteolysis in fibrin overcomes the limitations related to the deviation from the homogeneity assumptions by introducing a microscale stochastic approach, which operates with single enzyme molecules rather than enzyme concentrations in deterministic equations (16). This approach circumvents the necessity for assumptions of conventional transport phenomena, but its theoretical predictions for the efficiency of individual enzyme molecules have received only indirect support from experiments, because currently there are no direct data that track the behavior of separate molecules in the course of fibrinolysis. The present study was undertaken in 
attempt to combine the advantages of the existing kinetic models of fibrinolysis (operating with parameters based on easily accessible empirical data) and to minimize their limitations (adding mechanistic meaningfulness to these operational parameters based on nanoscale tracing of molecular events). Following the recent advances in single-molecule experimentation, significant effort is put at present to express the stochastic behavior of molecules in global kinetic rate equations (17) and thus our work translates this current focus of enzyme kinetics theory to the field of fibrinolysis developing a mechanistically validated tool for experimental investigation of the process.

Fractal kinetics provides a theoretically well-substantiated approach to describe chemical reactions under dimensional or topological constraints (18). According to this concept as a result of spatial restrictions in diffusion, the reactants cluster and segregate in the course of the reactions, which implies ageing of the reaction system (i.e. at identical instantaneous reactant concentrations at the time of observation, the reaction in an "older" system proceeds at a lower rate than in a "younger" one because of the more advanced degree of segregation) (18). This feature of fractal kinetics is reflected in the time-dependence of the instantaneous reaction rate coefficient $k$, which replaces the rate constant of classic kinetics $\left(k=k_{0}(1+t)^{-h}\right.$, where $k_{0}$ is the initial rate coefficient, $t$ is time and $h$ is a system-dependent exponent). As discussed above, fibrin forms multiple structured obstacles to fluid-borne enzymes and thus a fractal kinetic model could be potentially adequate to describe the plasmin-catalyzed degradation of fibrin. If the catalytic process is simplified to a three-step reaction scheme $E+F g_{R} \underset{k_{-1}^{F}}{\stackrel{k_{1,0}^{F}, h}{\rightleftarrows}} E F g \stackrel{k_{c a t}^{F}}{\longrightarrow} E+F D P_{t}$, where $E$ is plasmin, $F g_{R}$ is fibrin in the reactive interface and the rate coefficients of the separate reactions are indicated by $k$, the spatial constraints of the fibrin network affect only the diffusing reactant $(E)$ resulting in a time- 
dependent rate coefficient for the association of plasmin and fibrin $k_{1}^{F}=k_{1,0}^{F} /(t+1)^{h}$. Thus, if the steady state assumption is valid for discrete time intervals, the classical Michaelis-Menten equation developed originally for well-mixed homogeneous reaction systems (reviewed in 19)

can be applied in a modified form, in which the Michaelis constant $K_{m, 0}^{F}=\frac{k_{-1}^{F}+k_{c a t}^{F}}{k_{1,0}^{F}}$ is replaced by a time-dependent coefficient $K_{m}^{F}=K_{m, 0}^{F} \cdot(t+1)^{h}$. A similar modification of the MichaelisMenten model has been successfully applied in a different experimental setting (20). In the present study the analytical power of the fractal kinetic treatment of fibrinolysis has been tested on various fibrin structures and for known modifiers of plasmin action in order to establish its practical benefits.

\section{EXPERIMENTAL PROCEDURES}

Generation of plasmin- Plasmin was generated using plasminogen (isolated from human plasma activated by streptokinase (Calbiochem, LaJolla, CA, USA) at $172.5 \mathrm{U} / \mathrm{mg}$ zymogen. Plasmin concentrations were calculated by a method described elsewhere (21).

Turbidimetric fibrinolytic assay and initial data processing- Fibrin was prepared in microtiter plates from fibrinogen (human, plasminogen-depleted, Calbiochem) at concentrations $\left(F g_{0}\right)$ in the range $(1.5-5.9 \mu \mathrm{M})$ clotted by 5 or $100 \mathrm{nM}$ thrombin (thrombin from Serva Electrophoresis $\mathrm{GmbH}$ [Heidelberg, Germany] was further purified by ion-exchange chromatography on sulfopropyl-Sephadex yielding preparation with specific activity of $2100 \mathrm{IU} \mathrm{mg}$ (22) and 1 $\mathrm{IU} / \mathrm{mL}$ was considered equivalent to approximately $10.7 \mathrm{nmol} / \mathrm{L}$ by active site titration (23)) for $45 \mathrm{~min}$ at $37^{\circ} \mathrm{C}$ (in a total volume of $100 \mu \mathrm{l}$ in $10 \mathrm{mM}$ Hepes buffer $\mathrm{pH} 7.4$ containing $150 \mathrm{mM}$ 
$\mathrm{NaCl}$ and $3 \mathrm{mM} \mathrm{CaCl}_{2}$ ). Thereafter $60 \mu \mathrm{l}$ plasmin at concentrations $\left(E_{t 0}^{0}\right)_{\text {nominal }} 0.25-1 \mu \mathrm{M}$ was added to the surface of the clots followed by $60 \mu \mathrm{l}$ mineral oil layered over the solution to prevent evaporation. The course of clot dissolution was monitored by measuring the light absorbance $(A)$ at $340 \mathrm{~nm}$ at $37^{\circ} \mathrm{C}$. For the sake of data processing each unique pair of nominal initial plasmin $E_{t 0, j}^{0}$ and initial fibrin $F g_{0, j}$ concentrations was considered as a separate experiment with number $j=1,2, \ldots, J$ performed in $K_{j}$ replicas (typically 8). Within each experiment a linear dependence between the quantity of residual fibrin $(F)$ and the measured absorbance $(A)$ was assumed and normalization of $F$ was performed based on the initial and final values of $A$ as described in Supplemental Information. The mean value and the standard deviation of $F$ were calculated for each time point of each experiment and outliers were excluded based on criteria described in Supplemental Information. The heterogeneous surface at the boundary fibrin/fluid interface resulted in variability in the amount of fibrin-entrapped plasmin, which was independently estimated following withdrawal of the fluid phase-born plasmin after 15-min incubation over fibrin in the experimental setup described above and addition of $60 \mu \mathrm{l}$ $0.6 \mathrm{mM}$ Spectrozyme-PL (H-D-norleucyl-hexahydrotyrosyl-lysine-p-nitroanilide, American Diagnostica, Pfungstadt, Germany). The amount of fibrin-entrapped plasmin was reflected in the rate of hydrolysis of Spectrozyme-PL measured as changes in absorbance at $405 \mathrm{~nm}\left(\Delta \mathrm{A}_{405}\right)$. The uncertainties in the actual initial plasmin concentration $\left(E_{t, 0}^{0}\right)_{a c t u a l}$ were accounted for by the factor $C=\left(E_{t, 0}^{0}\right)_{\text {actual }} /\left(E_{t, 0}^{0}\right)_{\text {nominal }}$ with probability distribution calculated from measurements of the ratio $\left(\Delta \mathrm{A}_{405}\right.$ on fibrin surface/ $\Delta \mathrm{A}_{405}$ in fibrin-free system) and implemented in the kinetic model as described in Supplemental Information. 
Fractal kinetic model of the fibrinolytic process and estimation of its parameters- The Insets of Fig. 1 illustrate the notations used for the compartments and molecular species in the model of the experimental setup described above. The reaction proceeds at the interface of the two compartments with volume $V_{R}$, which is a sub-volume of $V_{d}$. The $V_{R}$ compartment moves down at the same rate as the rate of fibrin conversion to $F D P$. As a result part of the volume $V_{R}$ is continuously transforming into $V_{u}$ and $V_{R}$ contains constant $F g_{R t}$ (because of the progressive dissolution of fibrin, $F g_{R t}$ coincides with $F g_{0}$ ). In our model the plasmin-catalyzed degradation of fibrin in $V_{R}$ is described with the following scheme $E+F g_{R} \underset{k_{-1}^{F}}{\stackrel{k_{1,0}^{F}, h}{\rightleftarrows}} E F g \stackrel{k_{c a t}^{F}}{\longrightarrow} E+F D P_{t}$, where the rate coefficients of the separate reactions are indicated by $k$. The assumption for fractal kinetic behavior of plasmin under the spatial constrains of the fibrin network results in time dependence of the rate coefficient for the association of plasmin and fibrin $k_{1}^{F}=k_{1,0}^{F} /(t+1)^{h}$ and thus the Michaelis coefficient $K_{m}^{F}=\frac{k_{-1}^{F}+k_{c a t}^{F}}{k_{1}^{F}}$ will be also time-dependent $K_{m}^{F}=K_{m, 0}^{F} \cdot(t+1)^{h}$. The interaction of plasmin with fibrin degradation products is accounted for by a rapid equilibrium $E+F D P \underset{k_{-1}^{F D P}}{\stackrel{k_{1}^{F D P}}{\rightleftarrows}} E F D P$ characterized by $K_{a}^{F D P}=\frac{k_{1}^{F D P}}{k_{-1}^{F D P}}$ equilibrium binding constant yielding $E F D P=K_{a}^{F D P}$.E.FDP. For derivation of the kinetic equations of the model system the steady state assumption $\frac{d\left(E F g \cdot V_{R}\right)}{d t} \approx 0$ is used leading to $E F g=\frac{E \cdot F g_{R}}{K_{M}^{F}}$ and to the differential equation $\frac{d F D P_{t}}{d t}=k_{c a t}^{F} \cdot E F g \frac{V_{R}}{V_{u}+V_{R}}-F D P_{t} \frac{k_{c a t}^{F} \cdot E F g \cdot V_{R}}{F g_{0} \cdot\left(V_{u}+V_{R}\right)}$ for fibrin degradation as described in Supplemental Information. This equation is solved with initial condition $F D P_{t}(0)=0$ and based on $F D P_{t}, V_{d}$ is calculated as a function of time till $V_{d}$ reaches a value approaching $V_{R}$ 
by $0.5 \%$ (at this terminal stage residual fibrin is present only in a volume of $1.005 V_{R}$ ). Thus, finally the model function for residual fibrin is calculated $F=F_{m o d e l}\left(t,\left(E_{t, 0}^{0}\right)_{\text {actual }}, F g_{0}, K_{m, 0}^{F}, h, k_{\text {cat }}^{F}\right.$ ,$\left.K_{a}^{F D P}\right)=F g_{0 .} V_{d}(t)$. Because $\left(E_{t, 0}^{0}\right)_{a c t u a l}$ is a random variable with known distribution, the resulting $F_{\text {model }}$ is also a random variable with mean and standard deviation estimated using triple calculation of the derived $F_{m o d e l}$ function (see Supplemental Information). The parameters $K_{m, 0}^{F}$, $h, k_{c a t}^{F}, K_{a}^{F D P}$ were identified by a weighted least-square minimization of the $\chi^{2}$ statistic function as detailed in Supplemental Information. The confidence intervals of the optimal parameters are estimated with a multiplicative Monte Carlo simulation procedure which generates 150 synthetic data sets processed in the same way as the empirical data (the details of this approach are described in Supplemental Information). All programs for data processing, model simulation and parameter estimation described above run under Matlab 2013b (The MathWorks Inc., Natick, MA).

Scanning electron microscope (SEM) imaging of fibrin- Fibrinogen $(5.9 \mu \mathrm{M}$ in $25 \mathrm{mM} \mathrm{Na}-$ phosphate pH 7.4 buffer containing $75 \mathrm{mM} \mathrm{NaCl}$ ) was clotted for $1 \mathrm{~h}$ at $37^{\circ} \mathrm{C}$ with 5 or $100 \mathrm{nM}$ thrombin in Eppendorf tubes (pre-treated with $25 \mathrm{v} / \mathrm{v} \%$ Triton X-100 solution for $1 \mathrm{~h}$ and thoroughly washed with water). Clots were fixed, dehydrated, sputter coated with gold and examined as described in (15).

Atomic force microscopic (AFM) imaging of fibrin- Fibrin clots were prepared from $6.5 \mu \mathrm{M}$ fibrinogen clotted with $5 \mathrm{nM}$ thrombin for $30 \mathrm{~min}$ at room temperature on freshly cleaved mica sheets (Ted Pella Inc., CA) in a total volume of $50 \mu 1$ within $1 \mathrm{~cm}$ diameter rings enclosed by silicon grease. Plasmin at $1 \mathrm{nM}$ was added to the clots and after $20 \mathrm{~min}$ clots were washed with $100 \mathrm{mM}$ Na-cacodylate pH 7.2 buffer and fixated in $3 \%$ glutaraldehyde for $10 \mathrm{~min}$. Following 3 
washes with distilled water the samples were dehydrated in a $20-100 \%$ dilution series of acetone and following a 10-min treatment with hexamethyldisilizane (24) air-dried for at least 30 min. AFM images were taken with Multimode AFM head (DI, CA, USA) with Nanoscope V controller (Veeco, CA, USA) in contact mode in air at room temperature using a commercially available cantilever with a force constant of $0.12 \mathrm{~N} \mathrm{~m}^{-1}$ and a $\mathrm{Si}_{3} \mathrm{~N}_{4}$ tip (Veeco Nanoprobe, NP model).

Preparation of nanogold-labeled antibody- Labeling of anti-plasmin antibody (R\&D Systems, Abingdon, UK) was carried out according to published procedures (25). Briefly, sulfo-Nhydroxy-Succinimido $1.4 \mathrm{~nm}$ nanogold (Nanoprobes Inc., Yaphank, NY) reagent was dissolved in $200 \mu \mathrm{l}$ of deionized water immediately before use to give a $30 \mu \mathrm{M}$ solution. The anti-plasmin antibody was incubated with the activated nanogold solution for $1 \mathrm{~h}$ at room temperature (final concentrations: $11.5 \mu \mathrm{M}$ nanogold, $0.61 \mathrm{mg} / \mathrm{ml}$ anti-plasmin antibody). Conjugated and free nanogold were separated by fast pressure liquid chromatography using a Sephacryl S-200 HR column. Conjugated nanogold was collected and stored at $-20^{\circ} \mathrm{C}$ until use.

Preparation of patterned fibrinogen substrate surface with micro-contact printing and monitoring of plasmin distribution with AFM- Fibrinogen was printed on mica using microcontact printing $(25,26)$. A $15 \mathrm{~mm}$ diameter poly(dimethylsiloxane) (PDMS) (Sylgard 184, Dow Corning) rubber stamp patterned with pillar arrays of $0.7 \mu \mathrm{m}$ diameter was prepared by replication molding of silicon micropatterns prepared using optical lithography techniques. The PDMS stamp was incubated in $1 \mathrm{~g} / 1$ fibrinogen (in PBS: $0.01 \mathrm{M}$ sodium phosphate buffer, 150 $\mathrm{mM} \mathrm{NaCl}, \mathrm{pH}$ 7.4) for 1 hour; the stamp was then rinsed in PBS for 5 minutes, dipped in distilled water and dried with $\mathrm{N}_{2}$ flush. Freshly cleaved mica was used as the substrate for stamping experiments. The PDMS stamps were placed onto the mica, adequate stamping of 
proteins onto the substrate was ensured. The stamp was carefully peeled off thereafter, and the substrate surface was incubated in $1 \mathrm{~g} / 1$ bovine serum albumin (Sigma, Budapest, Hungary) in PBS for 1 hour to allow coating of areas of the surface not occupied by fibrinogen. Samples were incubated in 0.01 or $1 \mathrm{nM}$ plasmin in PBS. The reaction was stopped after 1 or 5 min by withdrawal of plasmin solution and dilution with PBS. Fixing was performed with $1 \mathrm{v} / \mathrm{v} \%$ glutaraldehyde in PBS for 10 min. Ethanolamine was applied for 10 min to eliminate unbound glutaraldehyde. The samples were then incubated in nanogold-labeled anti-plasmin antibody (diluted to $2 \mathrm{mg} / \mathrm{l}$ in PBS) for 1 hour at room temperature. Each of the above mentioned steps were followed by rinsing the sample in PBS for 5 minutes. Thereafter samples were examined with AFM as described above for fibrin imaging. Images were processed using NanoScope Analysis software 1.40 (Bruker, Germany) as described below.

Height analysis of AFM images of patterned fibrinogen surface and determination of bearing ratio- The average height of stamped fibrinogen layer was determined using height analysis of samples prior plasmin digestion. Bearing analysis was carried out on $1.5 \times 1.5 \mu \mathrm{m}$ regions of plasmin-treated samples, each containing a single square of printed fibrinogen, 27-54 regions per image at 4,500x magnification or 9 regions at 13,000x magnification (data analysis was carried out on 4 independent sets of experiments). To measure the percentage of surface area occupied by fibrinogen, bearing depth was set to $3 \mathrm{~nm}$ (based on results from control measurements). By setting the bearing depth value to $5 \mathrm{~nm}$, the percentage of area occupied by plasmin on the surface of fibrinogen is measured. To determine the occupancy of fibrinogen surface by plasmin, the fraction of fibrinogen surface area occupied by plasmin was calculated for each of the selected regions as ratio ( $5 \mathrm{~nm}$ bearing percentage $) /(3 \mathrm{~nm}$ bearing percentage $)$ and expressed as percentage. 
Cloning and expressing PLG_S195A_CFP-Full length cDNA for plasminogen was purchased from HGMP Resource Centre (IMAGE clone ID:4609295). The plasminogen coding region was amplified by PCR and ligated into pFastBac 1 (Life Technologies) to create pFastBac-PLG. A derivative of pFastBac-PLG was generated by site-directed mutagenesis (Stratagene) in which codon 195 of plasminogen was converted from AGT (serine) to GCT (alanine) to create pFastBac-PLG:S195A. pFastBac-PLG:S195A was modified to express a C-terminal fusion with enhanced cyan fluorescent protein (CFP) by modifying the plasminogen "stop" codon to an XbaI restriction site by site-directed mutagenesis. The XbaI fragment of pECFP (Clontech) that includes the coding sequence for CFP was ligated into the modified $X b a \mathrm{I}$ site to create $\mathrm{pFastBac-}$ PLG:S195A-CFP. The Bac-to-Bac baculovirus expression system (Invitrogen) was used to express PLG:S195A-CFP in Sf9 cells in serum-free SF900II media. PLG:S195A-CFP was secreted and purified from the culture media using a heparin agarose (Sigma) column for capture and concentration, eluting with $0.75 \mathrm{M} \mathrm{NaCl}$ on to a lysine-Sepharose (Sigma) column and eluted with $0.2 \mathrm{M}$ tranexamic acid (Sigma).

Confocal microscopic imaging of fibrin in the course of lysis- Fibrin clots were prepared from $6.5 \mu \mathrm{M}$ fibrinogen, $2 \%$ of which was Alexa Fluor ${ }^{\circledR}$ 546-conjugated fibrinogen (Invitrogen Life Technologies, Budapest, Hungary), clotted with $5 \mathrm{nM}$ thrombin for $30 \mathrm{~min}$ at room temperature in sterile, uncoated IBIDI VI $0.4 \mu$-slides (ibidi GmbH, Martinsried, Germany). Thereafter a mixture of $10 \mu \mathrm{g} / \mathrm{ml}$ plasminogen-S:A-CFP and $0.1 \mu \mathrm{M}$ plasmin was added to the edge of the clot and the fluorescence (excitation wavelength $458 \mathrm{~nm}$, emission wavelength $470 \mathrm{~nm}$ for plasminogen-S:A-CFP detection and excitation wavelength $543 \mathrm{~nm}$, emission wavelength 575 $\mathrm{nm}$ for Alexa546-fibrinogen detection) was monitored with Confocal Laser Scanning System LSM710 (Carl Zeiss GmbH) taking sequential images of the fluid-fibrin interface at a distance of 
approximately $50 \mu \mathrm{m}$ from the plate surface with identical exposures and laser intensities using a Plan-NeofluarX20/0.5 objective. All proteins were diluted in $25 \mathrm{mM} \mathrm{Na-phosphate} \mathrm{pH} 7.4$ buffer containing $75 \mathrm{mM} \mathrm{NaCl}$.

\section{RESULTS AND DISCUSSION}

Fractal kinetic treatment of progress curves in fibrinolysis- The turbidimetric assay (Fig. 1) is a robust experimental setup to acquire high number of data points for the resolution of preformed fibrin by fluid-phase borne plasmin. The optical signal in this assay is always proportional to the amount of intact fibrin with monomer concentration $\mathrm{Fg}_{0}$ in the volume $V_{d}$. The catalytic process occurs in the interface formed after the addition of plasmin at concentration $E_{t 0}^{0}$ in volume $V_{u}$. As a result of fibrin degradation and release of FDPs small enough not to produce any optical signal, $V_{d}$ continuously decreases and $V_{u}$ increases with consequent dilution of free plasmin in the bulk fluid phase. However, if the classic Michaelis-Menten model is applied for the catalytic events in the reactive layer $V_{R}$, this dilution factor cannot account for the deceleration trend in the progress curves of lysis (Fig. 1A); systematic deviations of the optimized model predictions from the measured values are seen at all stages of the process. Importantly, our numeric treatment of the model progress curves takes into account the variability in the plasmin concentration in the reactive layer through a randomizing factor based on independent measurements of plasmin activity retained at the fluid-fibrin interface, but even with this larger standard deviation (SD) allowance arising from the initial concentration uncertainties the values predicted by the Michaelis-Menten model differ significantly from the measured progress curve (see Supplemental Information, Fig. S11). Plasmin interaction with 
FDPs could also contribute to the declining speed of fibrinolysis, because binding of plasmin to these soluble products would withdraw protease molecules from the reactive interface layer. If this factor is taken into account in the model as a rapid equilibrium $E+F D P \underset{k_{-1}^{F D P}}{\stackrel{k_{1}^{F D P}}{\longrightarrow}} E F D P$ characterized by $K_{a}^{F D P}=\frac{k_{1}^{F D P}}{k_{-1}^{F D P}}$ equilibrium binding constant, the fitted model progress curves approach better the measured values (Fig. S11 in Supplemental Information), but the systematic deviations still persist, especially in the initial stage of the process. Significant improvement in the goodness-of-fit (reduction of $\mathrm{Chi}^{2}$ from 0.95 to 0.24 ) could be achieved with the fractal kinetic model (Fig. 1 B\&C). The time-dependent increase of the $K_{m}^{F}$ value of this model (Fig. 1D) in conjunction with the plasmin-FDP interaction satisfactorily describes the decline of the fibrinolytic rate in the complete course of the reaction.

Dependence of the fractal Michaelis coefficient on enzyme concentration- The applicability of the fractal kinetic model was tested under variable conditions for fibrinolysis. Varying the plasmin concentration in the physiologically relevant range between 0.25 and $1 \mu \mathrm{M}$, the $k_{c a t}^{F}$ parameter of the model remained constant, whereas the initial value of the $K_{m}^{F}$ coefficient was more than doubled (Table 1). Such a dependence of the apparent Michaelis constant on enzyme concentration in heterogeneous phase fibrinolysis is not unexpected in view of the recently developed generic rate law for surface-active enzymes (27). As elegantly demonstrated in this theoretical study, if a fluid-phase enzyme acts on a surface-bound substrate, the value of the Michaelis constant appears larger at increasing enzyme concentration, because the available area for absorption decreases monotonously when more enzyme is applied. Thus, our heterogeneous phase fibrinolytic assay provides empirical support for this theoretical prediction. The 
accompanying decrease in the fractal exponent $h$ of our model at higher plasmin concentration (Table 1) can be also attributed to the effect of available absorption area, which in the course of time poses constant limits to the clustering potential, whereas the fraction of uniformly dispersed enzyme is proportional to the total concentration. The phenomenological consequence is weaker time-dependence of $K_{m}^{F}$.

Fibrin structure as a modifier of the fractal kinetic behavior of plasmin- Fibrin is not a simple surface substrate; at identical monomer concentration various structures are formed depending on the polymerization conditions (Fig. 1D, Insets). As we and others have previously shown (4,5 and studies cited therein), fibrin formed at high thrombin concentration resulting in thin fibers is more difficult to dissolve on macroscopic scale than fibrin formed at low thrombin composed of thick fibers despite the faster digestion of individual thin fibers. This controversy is reflected in and explained by the values of the parameters of our present fractal kinetic model for the fibrin formed at 5 and $100 \mathrm{nM}$ thrombin (Table 1). The moderately (by $30 \%$ ) higher $k_{c a t}^{F}$ and two-fold lower initial $K_{m}^{F}$ values for the fine fibrin structure suggest more efficient proteolytic action of plasmin on this fibrin substrate. However, the overall time course of fibrinolysis is faster in the coarse fibrin at any examined fibrinogen concentration (compare Figs. 1B and C). In our fractal kinetic model this controversy is accounted for by the higher value of the fractal exponent $h$ in the thin-fiber substrate, which results in faster rise in the $K_{m}^{F}$ value (Fig. 1D) and thus deceleration of the lysis. According to the hypothesis for the 'crawling' action of plasmin (28) because of the closer distance of binding sites in the cross-section of the fibrin fibers $(6 \mathrm{~nm}$ on neighboring monomers in transverse section versus $22.5 \mathrm{~nm}$ in longitudinal direction along the protofibrils) plasmin moves preferentially in a lateral direction leading to transection of the individual fiber before dissociation and diffusion of the enzyme molecule to a remote site in the 
same or different fiber. Thus, plasmin cleaves the single thin fibers faster with fewer monomers in the cross-section, but with the progress of the lysis digestion becomes more dependent on the slower diffusion steps. In contrast, the larger mass within a single cross-section requires longer time for cleaving individual fibers in coarse fibrin, but because of the fewer fibers, the decelerating effect of the propagating diffusion-mediated jumps to remote fibers is less pronounced in this fibrin architecture (lower $h$ value, Table 1; slower increase of the $K_{m}^{F}$, Fig. 1D). Thus, the difference in the $h$ values for fine and coarse fibrin supports the mechanistic justification of the fractal exponent in the model as a coefficient reflecting the spatial redistribution of the enzyme.

Impact of modifiers of plasmin-fibrin interactions on the fractal kinetic parameters- We have recently shown that the continuous action of carboxypeptidase B (CPB) in lysing fibrin accelerates the plasmin-mediated fibrinolysis through removal of newly exposed C-terminal lysine residues (29). A comparable effect could be observed in a heterogeneous phase fibrinolytic assay in the presence of $1 \mathrm{mM} \varepsilon$-aminocaproic acid (EACA), whereas $\varepsilon A C A$ at concentrations above $5 \mathrm{mM}$ inhibits the plasmin action (Fig. 2A). The inhibiting effect of $\varepsilon A C A$ is in agreement with the known interference of this lysine analog with the lysine-dependent binding of plasmin to fibrin resulting in maximal inhibition of fibrin monomer digestion at $5 \mathrm{mM}$ concentration (7). However, neither the $\mathrm{CPB}$, nor the low-concentration $\varepsilon \mathrm{ACA}$ effects can be adequately treated in quantitative terms with classical enzyme kinetic models, whereas the fractal kinetic model could be successfully applied under these modified conditions too (Fig. 2B\&C). The time-dependent increase in the $K_{m}^{F}$ values was completely eliminated by both modifiers (observed as a decrease in the fractal exponent $h$ by at least an order of magnitude, Table 1), which suggests that the newly exposed C-terminal lysines in the course of lysis are primarily 
involved in the hypothesized progressive clustering of plasmin. The elimination of the redistribution-related ageing of the enzyme in the dynamically changing fibrin meshwork resulted in faster course of fibrinolysis either in CPB-modified fibrin or in the presence of $1 \mathrm{mM}$ \&ACA (however antagonizing higher affinity plasmin-fibrin interactions by $\varepsilon A C A$ at $5 \mathrm{mM}$ or higher concentration was inhibitory). It is noteworthy that in the presence of the accelerating concentration of $\varepsilon \mathrm{ACA}$ the $K_{m}$ not only lost its time dependence, but its value was higher than the initial $K_{m}^{F}$ in the modifier-free fibrinolytic assay (Table 1). No increase in $K_{m}$ was observed in the CPB-modified fibrin, which can be attributed to two opposing consequences of the CPB action. The removal of the C-terminal lysines exposed by plasmin would raise the $K_{m}$ similarly to EACA, but the presence of $\mathrm{CPB}$ at the stage of fibrin formation resulted in thinner fibers (e.g. 8 $\mathrm{U} / \mathrm{ml}$ CPB decreases the median fiber diameter in $6 \mu \mathrm{M}$ fibrin from $144 \mathrm{~nm}$ to $109 \mathrm{~nm}$ ) (29), a structure similar to the one formed by $100 \mathrm{nM}$ thrombin, in which plasmin operates with lower $K_{m}^{F}$ (Table 1). Thus, the effects of CPB on the parameters of the fractal kinetic model support the primary role of the progressive rise of $K_{m}^{F}$ in the relative lytic resistance of fibrin with fine structure; if this factor is eliminated by $\mathrm{CPB}$, plasmin acts with a constant lower $K_{m}$ and the overall course of lysis is significantly faster (Fig. 2A).

Structural evidence for progressive clustering of plasmin on fibrin surface- Because the key element of the fractal kinetic model discussed above is the clustering of plasmin molecules on the structured substrate surface, direct morphological evidence was also sought to substantiate this mechanism of action suggested by the macroscale course of fibrinolysis. On a microscopic scale, the distribution of plasmin on the surface of fibrin was monitored with the help of a fluorescent recombinant plasminogen variant plasminogen-S:A-CFP, which contains all fibrinbinding sites of plasmin, but cannot exert any catalytic activity with its active site serine replaced 
by alanine (Fig. 3). As a result of non-fluorescent plasmin action, new plasmin(ogen) binding sites become exposed in the reactive layer. This reorganization of the substrate surface altering the binding pattern of plasmin(ogen) is reflected in the behaviour of the fluorescent plasminogen variant. As shown in Fig. 3, although applied in a homogeneous solution to the surface of fibrin, following a 1 min digestion of fibrin with plasmin, plasminogen clusters were formed on the surface linked by a continuous layer of weaker plasminogen-related fluorescence. At a later stage of digestion the sites of the earlier clusters showed no detectable accumulation of plasminogen (arrows in Fig. 3), clusters were seen at different locations suggesting dynamic redistribution of plasmin(ogen) at the fluid-fibrin interface with increasing degree of heterogeneity. The focal nature of plasmin action was clearly demonstrated by the nanoscale traces in the AFM images of lysing fibrin (Fig. 4). However the same images also indicate that the random spatial arrangement of the fibrin fibers does not allow objective quantitation of the degree of heterogeneity of plasmin distribution on the surface of this network. To overcome this difficulty we designed a substrate surface for plasmin with structured pattern composed of fibrinogen arranged in well-defined squares (Fig. 5). Because of the regular pattern of the substrate, the relative area of intact fibrinogen covered by plasmin molecules could be reliably estimated based on the ratio of cross-section area at $5 \mathrm{~nm}$ height (corresponding to the nanogold-labeled antiplasmin antibody-enhanced plasmin on top of fibrinogen) and the cross-section area at $3 \mathrm{~nm}$ (corresponding to the height of the intact fibrinogen surface) shown as bearing ratio in Fig. 5B\&C. Visual inspection of the AFM images (Fig. 5A) evidences that at 1 min digestion, plasmin clusters were smaller in size and more uniformly dispersed over fibrinogen than at 5 min digestion, when larger clusters were preferentially localized at the edges of fibrinogen squares. Height analysis showed that this progressive clustering was not due to an absolute decrease in 
the fibrinogen area available for plasmin binding, but to redistribution of plasmin over the intact fibrinogen surface that could be quantitatively expressed as bearing ratio (Fig. 5B\&C). Decreasing relative occupancy of the intact substrate surface by plasmin is an indicator for loss of homogeneous distribution of the enzyme that occurs progressively in time (Fig. 5C) and is accelerated with the increase in enzyme concentration (Fig. 5B). Micro-contact printing creates a structured, heterogeneous surface for plasmin action. However, this method does not allow utilization of highly insoluble substances, e.g. fibrin. This is a limitation of the experimental system used here, because fibrinogen does not form polymers, and neither does it contain all plasmin(ogen) binding sites present in fibrin (30). With this limitation in mind, the dynamic clustering of plasmin on the patterned fibrinogen surface can be translated to the hypothetical situation in fibrin according to the scheme in Fig. 5D. At stage I (initial) the more uniformly dispersed enzyme and the larger available area allow for higher rate of association to fibrin reflected in lower initial values of $K_{m}^{F}$ (Fig. 1D). At stage II (late) the generated new plasmin binding sites (new C-terminal lysines) and the decreased available area (eliminated transected fibers) restrict the probability for new binding events seen as lower association rate and consequently higher $K_{m}^{F}$ values (Fig. 1D). As the lytic front moves, stage I state is formed in deeper clot layers and the relative expansion of stage II interfacial layer results in increase of $K_{m}^{F}$ in the course of time.

In conclusion, our study has substantiated in mechanistic terms a fractal kinetic model that describes satisfactorily the action of plasmin on fibrin surface. In this work earlier theoretical predictions for the behavior of surface-acting enzymes $(18,27)$ and qualitative evidence for spatially constrained migration of plasmin in the network of fibrin fibers (28) converge in a unified concept for quantitative characterization of interfacial fibrinolysis. This concept furthers 
our understanding of how various factors could affect in vivo fibrinolytic rates (as exemplified by the differential effects of fibrin structure on the separate parameters of the fractal kinetic model in this study). The fractal kinetic nature of the interfacial fibrinolysis adds a self-limiting factor to the action of plasmin, which is complementary to the plasma inhibitor-dependent decay of its activity in vivo and thus contributes to the higher sensitivity of the fibrinolytic system to regulation at the level of plasminogen activation that supplies new plasmin responding to system demands. In addition to the improved quantitative interpretation of physiological fibrinolysis, this model delineates novel strategies in the ongoing research of direct fibrinolytic enzymes (6). For example, it predicts that the catalytic efficiency of a protease acting on the surface of fibrin would benefit from retarded clustering of the enzyme, which could be achieved with recombinant modifications of the parent plasmin molecule (optimizing the number of its fibrinbinding sites and their fibrin affinity based on testing of enzyme variants in the newly introduced kinetic model). An alternative strategy could be the development of plasminogen activators that occupy plasmin binding sites in fibrin and consequently combine the advantage of local generation of plasmin in therapeutic thrombolysis with prevention of plasmin clustering. Thus, this model could contribute to the rational design of better thrombolytic agents to combat cardioand cerebrovascular disease, the major morbidity and mortality cause in the world.

\section{ACKNOWLEDGMENT}

The authors are grateful to Péter Fürjes for the help in silicon master preparation and to Györgyi Oravecz for technical assistance. 


\section{Supporting Information Available}

1. Experimental setup of the turbidimetric fibrinolytic assay, measurement and initial data processing

2. Model of a single progress curve

3. Identification of optimal parameters

4. Monte Carlo procedure for confidence intervals of the optimal parameters

5. Comparison of the goodness-of-fit of three kinetic models for the action of plasmin on fibrin surface

This material is available free of charge via the Internet at http://pubs.acs.org

\section{REFERENCES}

1. Armstrong, P.W., Gershlick, A.H., Goldstein, P., Wilcox, R., Danays, T., Lambert, Y., Sulimov, V., Rosell Ortiz, F., Ostojic, M., Welsh, R.C., Carvalho, A.C., Nanas, J., Arntz, H.R., Halvorsen, S., Huber, K., Grajek, S., Fresco, C., Bluhmki, E., Regelin, A., Vandenberghe, K., Bogaerts, K., Van de Werf, F., and STREAM Investigative Team (2013) Fibrinolysis or primary PCI in ST-segment elevation myocardial infarction, $N$. Engl. J. Med. 368, 1379-1387.

2. Adams, H.P. Jr., del Zoppo, G., Alberts, M.J., Bhatt, D.L., Brass, L., Furlan, A., Grubb, R.L., Higashida, R.T., Jauch, E.C., Kidwell, C., Lyden, P.D., Morgenstern, L.B., Qureshi, A.I., Rosenwasser, R.H., Scott, P.A., and Wijdicks, E.F. (2007) Guidelines for the early management of adults with ischemic stroke. Circulation 115, e478-e534. 
3. Kolev, K., Longstaff, C., and Machovich, R. (2005) Fibrinolysis at the fluid-solid interface of thrombi. Curr. Med. Chem. Cardiovasc. Hematol. Agents 3, 341-355.

4. Collet, J.P., Park, D., Lesty, C., Soria, J., Soria, C., Montalescot, G., and Weisel, J.W. (2000) Influence of fibrin network conformation and fibrin fiber diameter on fibrinolysis speed: dynamic and structural approaches by confocal microscopy. Arterioscler. Thromb. Vasc. Biol. 20, 1354-1361.

5. Longstaff, C., Thelwell, C., Williams, S.C., Silva, M.M., Szabó, L., and Kolev, K. (2011) The interplay between tissue plasminogen activator domains and fibrin structures in the regulation of fibrinolysis: kinetic and microscopic studies. Blood 117, 661-668.

6. Marder, V.J. (2011) Historical perspective and future direction of thrombolysis research: the re-discovery of plasmin. J. Thromb. Haemost. 9(S1), 364-373.

7. Kolev, K., Tenekedjiev, K., Komorowicz, E., and Machovich, R (1997) Functional evaluation of the structural features of proteases and their substrate in fibrin surface degradation. J. Biol. Chem. 272, 13666-13675.

8. Diamond, S.L., and Anand, S. (1993) Inner clot diffusion and permeation during fibrinolysis. Biophys. J. 65, 2622-2643.

9. Anand, S., Wu, J.H., and Diamond, S.L. (1995) Enzyme-mediated proteolysis of fibrous biopolymers: Dissolution front movement in fibrin or collagen under conditions of diffusive or convective transport. Biotechnol. Bioeng. 48, 89-107.

10. Wootton, D.M., Popel, A.S., and Alevriadou, B.R. (2002) An experimental and theoretical study on the dissolution of mural fibrin clots by tissue-type plasminogen activator. Biotechnol. Bioeng. 77, 405-419. 
11. Carr, M.E. Jr., and Hermans, J. (1978) Size and density of fibrin fibers from turbidity. Macromolecules 11, 46-50.

12. Voter, W.A., Lucaveche, C., and Erickson, H.P. (1986) Concentration of protein in fibrin fibers and fibrinogen polymers determined by refractive index matching. Biopolymers 25, 2375-2384.

13. Dix, J.A., and Verkman, A.S. (2008) Crowding effects on diffusion in solutions and cells. Annu. Rev. Biophys. 37, 247-263.

14. Verkman, A.S. (2013) Diffusion in the extracellular space in brain and tumors. Phys. Biol. 10, 045003

15. Varjú, I., Sótonyi, P., Machovich, R., Szabó, L., Tenekedjiev, K., Silva, M.M., Longstaff, C., and Kolev, K. (2011) Hindered dissolution of fibrin formed under mechanical stress. J. Thromb. Haemost. 9, 979-986.

16. Bannish, B.E., Keener, J.P., and Fogelson, A.L. (2014) Modelling fibrinolysis: a 3D stochastic multiscale model. Math. Med. Biol. 31, 17-44.

17. Xie, X.S. (2013) Enzyme kinetics, past and present. Science 342, 1457-1459.

18. Kopelman, R. (1988) Fractal reaction kinetics. Science 241, 1620-1626.

19. Cornish-Bowden, A. (2013) The origins of enzyme kinetics. FEBS Lett. 587, 2725-2730.

20. Kosmidis, K., Karalis, V., Argyrakis, P., and Macheras, P. (2004) Michaelis-Menten kinetics under spatially constrained conditions: application to mibefradil pharmacokinetics. Biophys. J. 87, 1498-1506.

21. Kolev, K., Léránt, I., Tenekejiev, K., and Machovich, R. (1994) Regulation of fibrinolytic activity of neutrophil leukocyte elastase, plasmin, and miniplasmin by plasma protease inhibitors. J. Biol. Chem. 269, 17030-17034. 
22. Lundblad, R.L., Kingdon, H.S., and Mann, K.G. (1976) Thrombin. Meth. Enzymol. 45, $156-176$.

23. Longstaff, C., Wong, M.Y., and Gaffney, P.J. (1993) An international collaborative study to investigate standardisation of hirudin potency. Thromb. Haemost. 69, 430-435.

24. Ubero-Pascal, N., Fortuño, J.M., and de Los Angeles, P.M. (2005) New application of air-drying techniques for studying Ephemeroptera and Plecoptera eggs by scanning electron microscopy. Microsc. Res. Tech. 68, 264-271.

25. Soman, P., Rice, Z., and Siedlecki, C.A. (2008) Immunological identification of fibrinogen in dual-component protein films by AFM imaging. Micron 39, 832-842.

26. Agnihotri, A., and Siedlecki CA (2005) Adhesion mode atomic force microscopy study of dual component protein films. Ultramicroscopy 102, 257-268.

27. Kartal, O., and Ebenhöh, O. (2013) A generic rate law for surface-active enzymes. FEBS Lett. 587, 2882-2890.

28. Weisel, J.W., Veklich, Y., Collet, J.P., and Francis, C.W. (1999) Structural studies of fibrinolysis by electron and light microscopy. Thromb. Haemost. 82, 277-282.

29. Kovács, A., Szabó, L., Longstaff, C., Tenekedjiev, K., Machovich, R., and Kolev, K. (2014) Ambivalent roles of carboxypeptidase B in the lytic susceptibility of fibrin. Thromb. Res. 133, 80-87.

30. Medved, L., and Nieuwenhuizen, W. (2003) Molecular mechanisms of initiation of fibrinolysis by fibrin. Thromb. Haemost. 89, 409-419. 


\section{TABLES}

Table 1. Fractal kinetic parameters of plasmin on fibrin meshwork substrate ${ }^{\alpha}$

\begin{tabular}{|c|c|c|c|c|c|c|}
\hline modifier $^{\beta}$ & $\begin{array}{l}\text { plasmin } \\
(\mu \mathrm{M})\end{array}$ & $\begin{array}{l}K_{m}^{F} \text { or } K_{m} \\
(\mu \mathrm{M})^{\gamma}\end{array}$ & $h(-)$ & $k_{c a t}^{F}\left(\min ^{-1}\right)$ & $K_{a}^{F D P}\left(\mu \mathrm{M}^{-1}\right)$ & $C h i^{2}$ \\
\hline none & 0.25 & $\begin{array}{l}2.13 \\
(1.90-2.45)\end{array}$ & $\begin{array}{l}0.25 \\
(0.24-0.26)\end{array}$ & $\begin{array}{l}32.31 \\
(32.28-32.35)\end{array}$ & $\begin{array}{l}0.44 \\
(0.37-0.58)\end{array}$ & 0.24 \\
\hline & 1.0 & $\begin{array}{l}5.01 \\
(4.96-6.03)\end{array}$ & $\begin{array}{l}0.16 \\
(0.12-0.17)\end{array}$ & $\begin{array}{l}32.31 \\
(32.11-32.32)\end{array}$ & $\begin{array}{l}0.84 \\
(0.77-1.03)\end{array}$ & 0.45 \\
\hline Th100 & 1.0 & $\begin{array}{l}1.98 \\
(1.80-2.22)\end{array}$ & $\begin{array}{l}0.25 \\
(0.23-0.27)\end{array}$ & $\begin{array}{l}42.41 \\
(42.39-42.42)\end{array}$ & $\begin{array}{l}0.43 \\
(0.39-0.48)\end{array}$ & 0.24 \\
\hline $\begin{array}{l}1 \mathrm{mM} \\
\varepsilon \mathrm{ACA}\end{array}$ & 0.25 & $\begin{array}{l}8.44 \\
(8.09-9.23)\end{array}$ & $\begin{array}{l}0.03 \\
(0.02-0.06)\end{array}$ & $\begin{array}{l}39.23 \\
(39.07-39.33)\end{array}$ & $\sim 0$ & 0.46 \\
\hline & 1.0 & $\begin{array}{l}7.52 \\
(7.45-7.69)\end{array}$ & $\sim 0$ & $\begin{array}{l}39.89 \\
(39.85-39.90)\end{array}$ & $\begin{array}{l}1.63 \\
(1.42-1.89)\end{array}$ & 0.51 \\
\hline $\mathrm{CPB}$ & 0.25 & $\begin{array}{l}2.68 \\
(2.39-2.84)\end{array}$ & $\sim 0$ & $\begin{array}{l}41.76 \\
(41.74-41.78)\end{array}$ & $\begin{array}{l}1.20 \\
(1.11-1.34)\end{array}$ & 0.54 \\
\hline & 1.0 & $\begin{array}{l}3.25 \\
(2.59-4.03)\end{array}$ & $\sim 0$ & $\begin{array}{l}41.63 \\
(40.81-45.42)\end{array}$ & $\begin{array}{l}1.04 \\
(0.65-1.65)\end{array}$ & 0.53 \\
\hline
\end{tabular}

${ }^{\alpha}$ Kinetic parameters are reported as best-fitted values and their $95 \%$ confidence intervals from 150 cycles of Monte Carlo simulations using a global fitting procedure to measurements illustrated in Figure 1. 
${ }^{\beta}$ Fibrin was prepared from fibrinogen at different concentrations clotted with $5 \mathrm{nM}$ thrombin (or $100 \mathrm{nM}$ thrombin indicated as Th100). For evaluation of the effect of $\varepsilon$-aminocaproic acid ( $\varepsilon A C A)$ and carboxypeptidase B (CPB) CPB was mixed with fibrinogen at $8 \mathrm{U} / \mathrm{ml}$ prior clotting, whereas $\varepsilon A C A$ was added together with plasmin at the surface of the clot.

$\gamma$ If a fractal kinetic model was used $(h \neq 0)$, the values of $K_{m}^{F}$ are reported for time $t=0$.

\section{FIGURE LEGENDS}

Figure 1. Optimized model and measured progress curves of fibrin degradation by plasmin. Fibrinogen at the indicated concentrations was clotted by 5 (panel B) or $100 \mathrm{nM}$ (panels A and C) thrombin for $45 \mathrm{~min}$, thereafter $1 \mu \mathrm{M}$ plasmin was added to the surface of fibrin and the absorbance at $340 \mathrm{~nm}$ was continuously monitored. The amount of intact fibrin $F n=F g_{0} . V_{d}$ was calculated from the absorbance data and shown as mean (symbols) and SD (continuous vertical lines) from 8 measurements. The model progress curves for parameters optimized according the classic Michaelis-Menten model (panel A) or the fractal kinetic model (panels B and C) are presented as continuous lines, whereas the model SD is indicated by vertical dashed lines. $C h i^{2}$ is the quantitative indicator for the goodness-of-fit as defined in the Supplemental Information. Panel D shows the time dependence of the plasmin $K_{m}{ }^{F}$ predicted by the fractal kinetic model for fibrin substrate prepared from fibrinogen clotted by 5 (dotted line) or 100 (solid line) nM thrombin, the structure of which is illustrated by the inset scanning electron microscope images (in both cases fibrinogen was at $5.9 \mu \mathrm{M}$, scale bar=2 $\mu \mathrm{m}$ ). Insets in panels A-C show the compartmentation of molecular species assumed for the respective models. $V_{d}$ is the volume occupied by intact fibrin at monomer concentration $F g_{0}$. In the reactive interface layer with volume $V_{R} F g_{0}$ is distributed between free fibrin $F g_{R}$ and plasmin-fibrin complex $E F g$. The $E_{t 0}$ is distributed among free plasmin $E$, plasmin bound to soluble fibrin degradation products $E F D P$ and $\mathrm{EFg}$. 
Figure 2. Effect of carboxypeptidase B and $\varepsilon A C A$ on plasmin-catalyzed fibrinolysis. A: Fibrinogen at $2.9 \mu \mathrm{M}$ was clotted with $5 \mathrm{nM}$ thrombin, after $45 \mathrm{~min} 1 \mu \mathrm{M}$ plasmin was added to the surface of the clot and the absorbance at $340 \mathrm{~nm}$ was measured (mean of 8 progress curves is shown). Carboxypeptidase B (CPB) was added to fibrinogen prior clotting, whereas \&ACA was present only in the plasmin solution. Measured (symbols) and model progress curves of lysis (continuous lines) are shown for CPB-modified fibrin (panel B) and $1 \mathrm{mM}$ EACA applied together with plasmin (panel C). The best fit for the lysis in B and C was achieved according to a non-fractal model including a rapid equilibrium term for the interaction of plasmin and FDPs. Vertical bars indicate the SD of the measurement (continuous lines) and the model (dashed lines).

Figure 3. Microscopic clustering of plasminogen in the lytic interfacial layer of fibrin. Alexa543-labeled fibrin was prepared as described in Experimental procedures and $0.1 \mu \mathrm{M}$ plasmin containing plasminogen-S:A-CFP was added to its surface. Images were taken in a confocal laser microscope 1 and 5 min after initiation of lysis. The plasminogen-S:A-CFP molecules are forming clusters along the lysis front, connected by a continuous layer of weaker fluorescent signal at $1 \mathrm{~min}$, which is interrupted at the sites indicated by arrows at $5 \mathrm{~min}$. Red channel: Alexa543-labeled fibrin (excitation $543 \mathrm{~nm}$, emission $575 \mathrm{~nm}$ ). Green channel: plasminogen-S:A-CFP (excitation $458 \mathrm{~nm}$, emission $470 \mathrm{~nm}$ ). Scale bar=50 $\mu \mathrm{m}$.

Figure 4. Focal nanoscale traces of plasmin action in three-dimensional fibrin meshwork. Fibrin was prepared on mica sheets and AFM images were taken as described in Methods. The continuity of the original fibrin fibers (left panel) is interrupted by transections (arrow) leading to 
extensive moth-hole-like digestive foci $20 \mathrm{~min}$ after application of $1 \mathrm{nM}$ plasmin to the clot (right panel).

Figure 5. Distribution of plasmin molecules on patterned fibrinogen substrate surface. Square $(0.7 \times 0.7 \mu \mathrm{m})$ fibrinogen islets of $3 \mathrm{~nm}$ height were prepared on mica sheets using micro-contact printing technique (A, left panel) and following plasmin treatment, stopping the digestion, glutaraldehyde fixation and exposure to nanogold-labeled anti-plasmin antibody AFM images were taken as described in Methods. A: representative images of plasmin-anti-plasmin complexes attached to fibrinogen digested for $1 \mathrm{~min}$ and $5 \mathrm{~min}$ with $1 \mathrm{nM}$ plasmin, scale bar=1.5 $\mu \mathrm{m}$. B and C: The bearing ratio representing the percentage of intact fibrinogen surface occupied by plasmin was determined on at least 39 AFM images of fibrinogen islets from 2 independent series of measurements for each type of samples ( $p$ values refer to Kolmogorov-Smirnov test executed on the indicated pairs of samples). The digestion time was $5 \mathrm{~min}$ in panel $\mathrm{B}$, the plasmin concentration was $1 \mathrm{nM}$ in panel C. D: Based on the results in panels A-C a scheme is proposed for the distribution of plasmin (red dots) across a cross-section of a fibrin clot in the plane of the reactive interface defined in Fig. 1 at an early (stage I) and late (stage II) time point of lysis (only fibrin fibers perpendicular to the interfacial plane are shown as green circles, assuming that their transection results in FDP release). In stage II the transected thinner are absent from the plane of the section, whereas the plasmin molecules form clusters, the sites of which can be traced back to the loci of digestion observed in the AFM images (Fig. 4). 

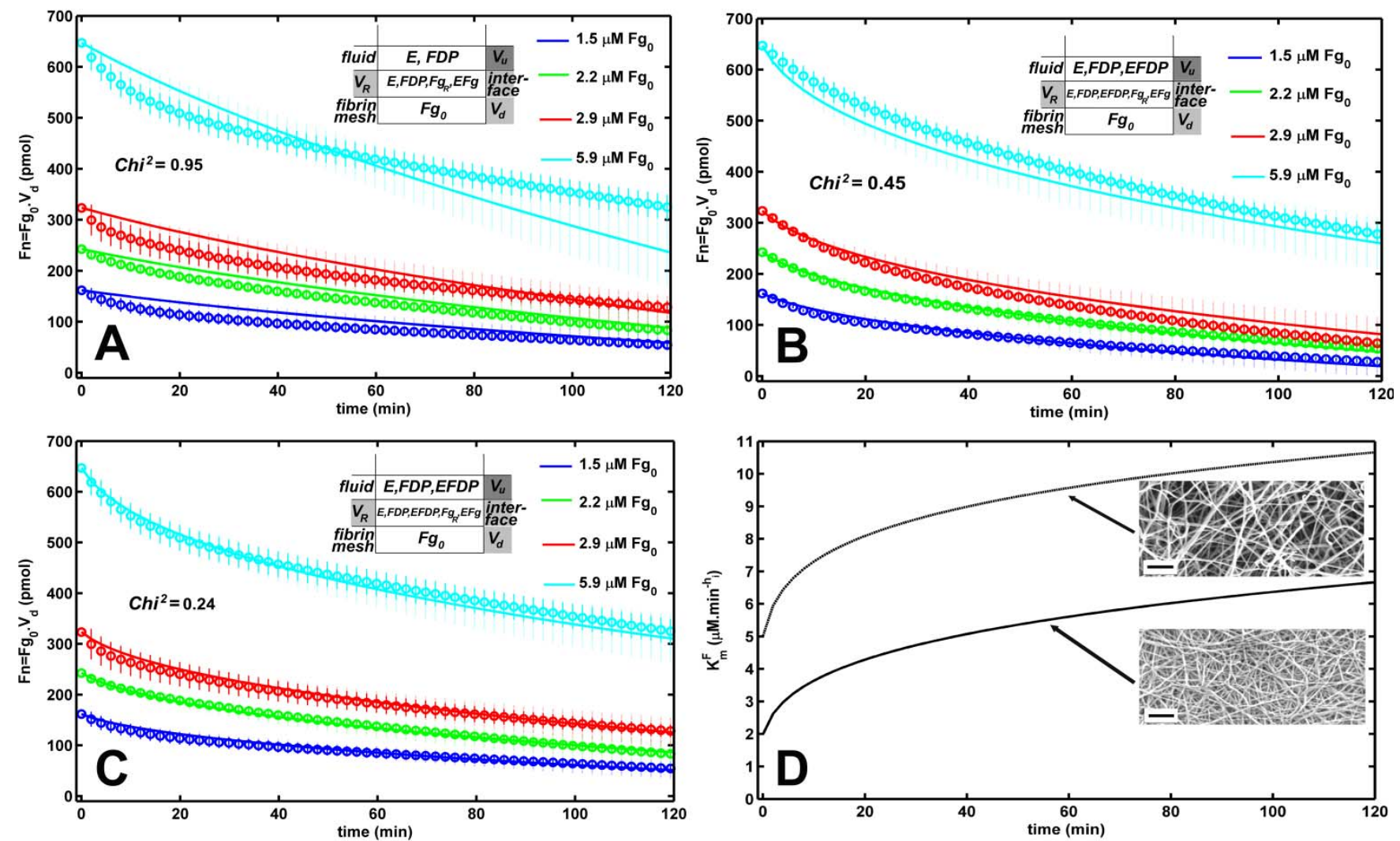

Figure 1
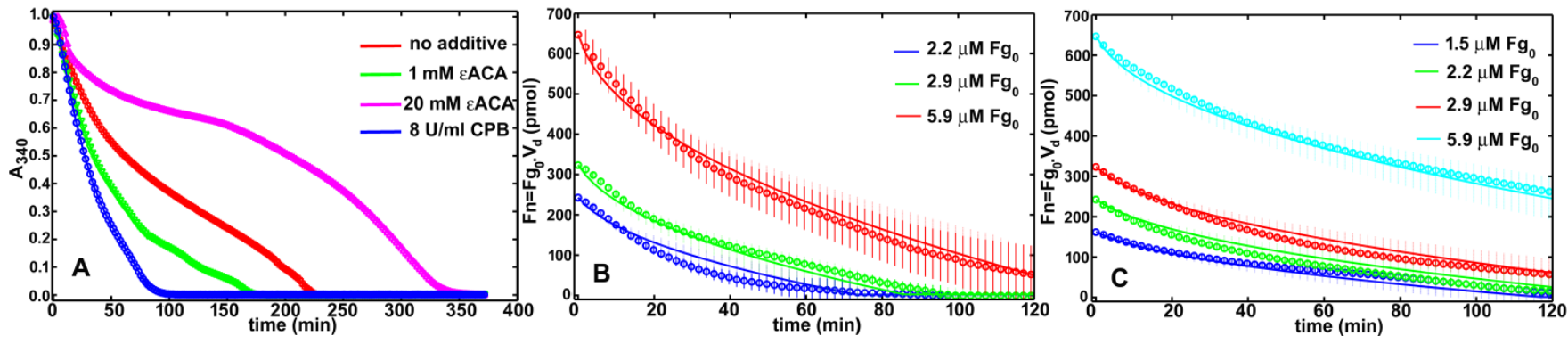

Figure 2
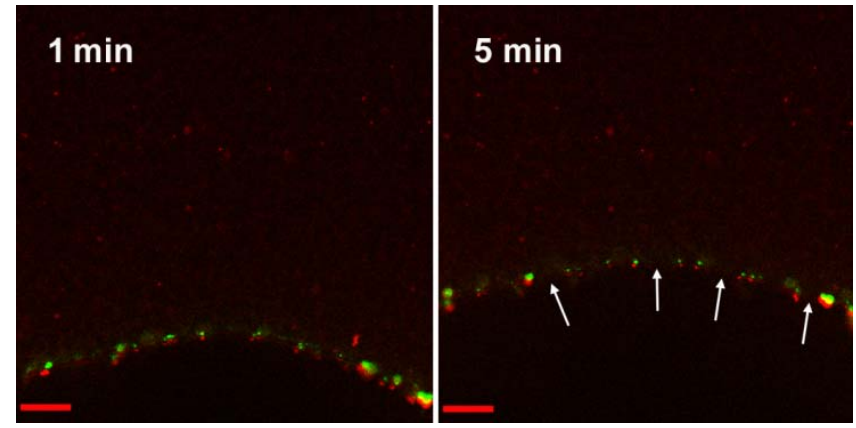

Figure 3 


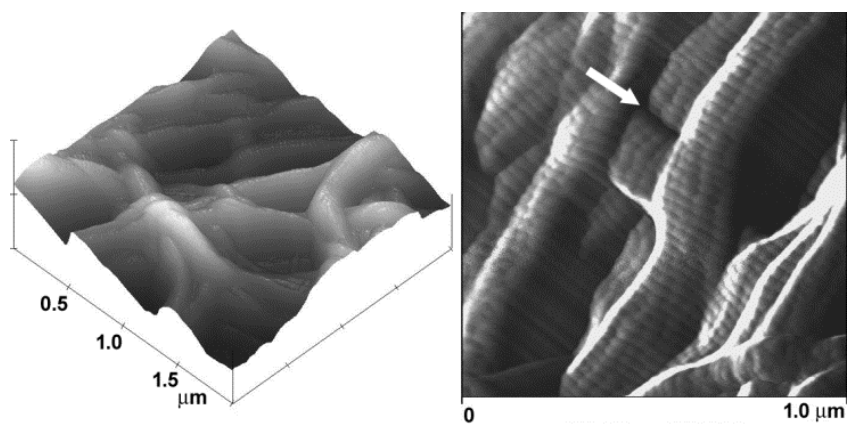

Figure 4

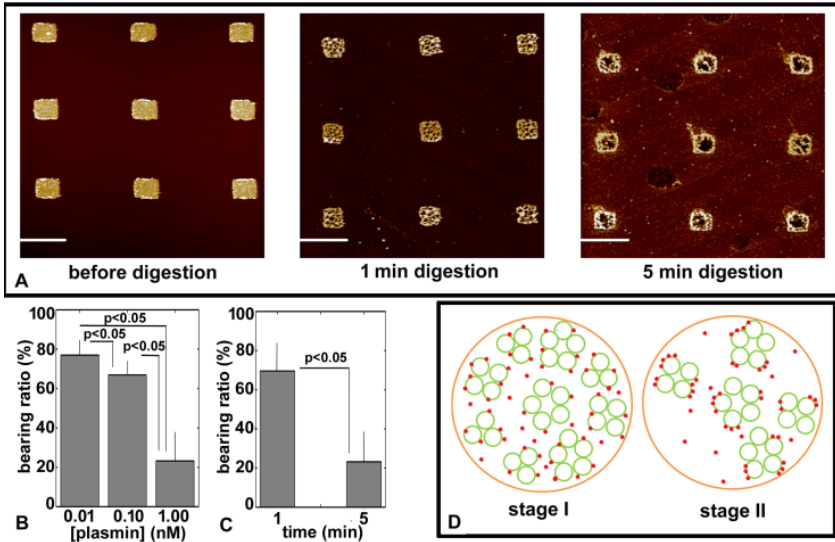

Figure 5 


\section{For Table of Contents Use Only}

Fractal Kinetic Behavior of Plasmin on the Surface of Fibrin Meshwork

Imre Varjú, Kiril Tenekedjiev, Zsófia Keresztes, Andrea Edit Pap, László Szabó, Craig Thelwell, Colin Longstaff, Raymund Machovich, Krasimir Kolev

Fibrinogen islets

